\title{
Star formation in a diffuse high-altitude cloud?
}

\author{
J. Kerp, D. Lenz, and T. Röhser
}

\begin{abstract}
Argelander-Institut für Astronomie Auf dem Hügel 71, 53121 Bonn, Germany
\end{abstract}
e-mail: jkerp@astro.uni-bonn.de

Received 17 July 2015 / Accepted 2 March 2016

\begin{abstract}
Context. A recent discovery of two stellar clusters associated with the diffuse high-latitude cloud HRK 81.4-77.8 has important implications for star formation in the Galactic halo.

Aims. We derive a plausible distance estimate to HRK 81.4-77.8 primarily from its gaseous properties.

Methods. We spatially correlate state-of-the-art HI, far-infrared and soft X-ray data to analyze the diffuse gas in the cloud. The absorption of the soft X-ray emission from the Galactic halo by HRK 81.4-77.8 is used to constrain the distance to the cloud.

Results. HRK 81.4-77.8 is most likely located at an altitude of about $400 \mathrm{pc}$ within the disk-halo interface of the Milky Way Galaxy. The HI data discloses a disbalance in density and pressure between the warm and cold gaseous phases. Apparently, the cold gas is compressed by the warm medium. This disbalance might trigger the formation of molecular gas high above the Galactic plane on pc to sub-pc scales.
\end{abstract}

Key words. Galaxy: halo - ISM: clouds - ISM: individual objects: HRK81.4-77.8 - stars: formation

\section{Introduction}

Camargo et al. (2015) report on the discovery of two embedded stellar clusters proposed to be physically associated with the high Galactic latitude cloud HRK 81.4-77.8 (or Heiles et al. 1988, G 81.4-77.8). Using WISE data (Wright et al. 2010) Camargo et al. (2015) deduce from their color-magnitude diagrams (CMDs) an age of about $2 \mathrm{Myr}$ for both stellar clusters, Camargo 438 and Camargo 439. Based on the absolute stellar luminosity, a distance from the Sun of about $5 \mathrm{kpc}$ is calculated. Because of their location close to the southern Galactic pole they are positioned nearly at the solar circle, but at an altitude of about $4 \mathrm{kpc}$ below the Galactic plane. Moreover, Camargo et al. (2015) identified two cluster member stars with known proper motions towards Camargo 439. This information is used to estimate the orbital parameters of the extra-planar clusters.

Our aim is to explore the physical conditions and the interstellar gas properties of HRK 81.4-77.8. So far only two high Galactic latitude clouds that form stars at altitudes of a few tens of pc above the Galactic plane, namely MBM 12 and MBM 20 (Magnani et al. 1985), have been identified (see McGehee 2008). Both star-forming MBM clouds are classified as dark interstellar clouds. Dark interstellar clouds show up with an optical extinction in excess of $A_{\mathrm{V}}=5 \mathrm{mag}$ (McGehee 2008).

We use state-of-the-art multifrequency data for our investigation. The HI $21 \mathrm{~cm}$ line data of the Parkes Galactic All-Sky Survey (GASS; Kalberla \& Haud 2015; Kalberla et al. 2010; McClure-Griffiths et al. 2009) and the Planck far-infrared (FIR) data (Planck Collaboration IX 2014) are analyzed to quantify the amount of molecular gas in HRK 81.4-77.8. The interstellar extinction is evaluated by the updated (Schlafly \& Finkbeiner 2011) Schlegel, Finkbeiner \& Davis (SFD) data set (Schlegel et al. 1998). ROSAT all-sky survey data (Snowden \& Schmitt 1990; Snowden et al. 1997) are analyzed to evaluate the soft X-ray shadow of HRK 81.4-77.8 allowing to estimate its distance from the Sun. Section 2 comprises the details of our analyses, while Sect. 3 contains our conclusions.

\section{Gaseous properties of HRK 81.4-77.8}

\subsection{HRK 81.4-77.8: A diffuse infrared cirrus cloud}

Using the improved SFD data set (Schlegel et al. 1998; Schlafly \& Finkbeiner 2011) on interstellar extinction HRK 81.4-77.8 reveals a maximum of only $A_{\mathrm{V}}=0.144 \mathrm{mag}$. According to this value it has to be classified as a diffuse cloud (McGehee 2008). Diffuse interstellar clouds are not expected to efficiently shield molecules against dissociation by high-energy photons, even within their densest portions. Thus, significant fractions of molecular gas are not expected to be present under these environmental conditions (Kennicutt \& Evans 2012). Hence, the physical association of the stellar clusters Camargo 438 and 439 with HRK 81.4-77.8 is a striking proposal.

\subsection{HRK 81.4-77.8: Distance estimate derived from interstellar reddening}

Very recently Green et al. (2015) published a 3D database of interstellar dust reddening based on optical and near-infrared surveys. Inspecting this data cube for the extinction towards HRK 81.4-77.8 discloses a faint signal. The best fit extinction shows a continuous increase in the reddening up to a distance of $400 \mathrm{pc}$. Beyond that distance the reddening remains constant out to $15 \mathrm{kpc}$. When HRK 81.4-77.8 is located at a distance of $5 \mathrm{kpc}$ we would expect a second increase in the reddening at this particular distance, which is not observed. Accordingly, the Green et al. (2015) database is not consistent with a location of HRK 81.4-77.8 at $5 \mathrm{kpc}$.

\subsection{Estimating the distance to HRK 81.4-77.8: ROSAT All-Sky Survey data}

Soft X-rays with photon energies below the carbon edge of $0.284 \mathrm{keV}$ are very sensitive to photoelectric absorption by the interstellar gas (Wilms et al. 2000). 

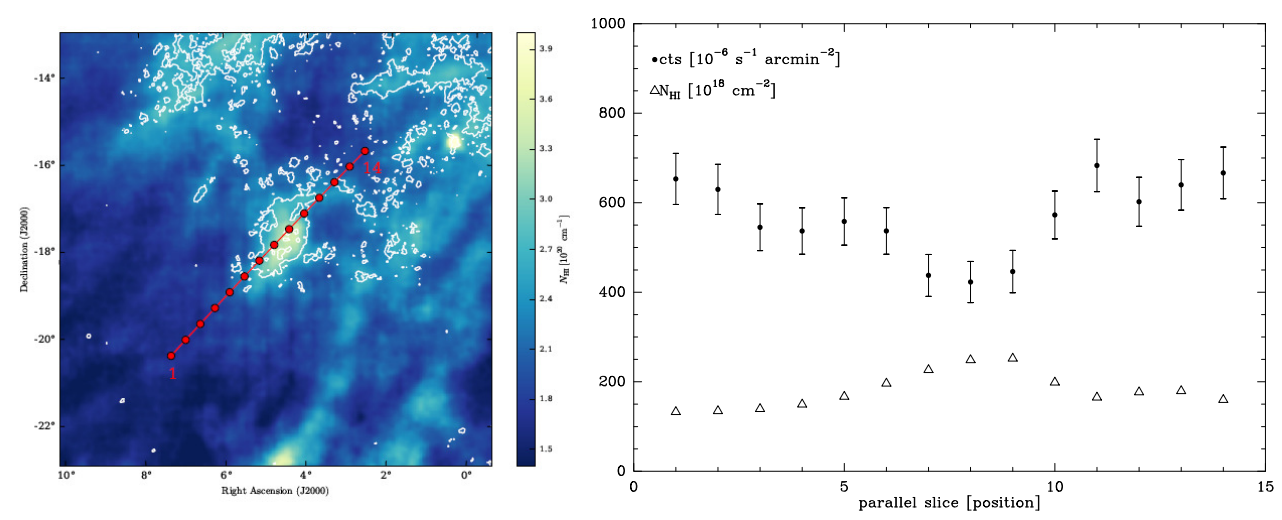

Fig. 1. Left: integrated $(-37.3 \leq$ $\left.v_{\text {LSR }}\left[\mathrm{km} \mathrm{s}^{-1}\right] \leq 52.9\right)$ GASS HI column density map of HRK 81.4-77.8. Superposed as contours is the Planck $857 \mathrm{GHz}$ intensity distribution starting at $0.76 \mathrm{MJy} \mathrm{sr}^{-1}$ in steps of $0.45 \mathrm{MJy} \mathrm{sr}^{-1}$. Right: slice across the longest extent of HRK 81.4-77.8 (see left panel, dots along the solid line). The open triangles mark the HI column density distribution $N_{\mathrm{HI}}$ in $10^{18} \mathrm{~cm}^{-2}$, the filled circles represent the ROSAT R12 count rate in $10^{-6} \mathrm{cts} \mathrm{s}^{-1} \operatorname{arcmin}^{-2}$.

The effective photoelectric absorption cross section at a column density of approximately $N_{\mathrm{HI}}=3.0 \times 10^{20} \mathrm{~cm}^{-2}$ is $\bar{\sigma} \simeq 0.8 \times 10^{-20} \mathrm{~cm}^{2}$ for a thermal plasma of $T=10^{6.2} \mathrm{~K}$ (see Snowden et al. 1994, Fig. 4). Thus, even diffuse interstellar gas at high galactic latitude modulates the soft X-ray background intensity distribution significantly.

Prior to the ROSAT mission (Snowden \& Schmitt 1990; Snowden et al. 1997) it was thought that the observed soft X-ray background emission originates entirely from a thermal plasma (Snowden et al. 1990) filling the local cavity (Frisch \& York 1983; Paresce 1984; Lallement et al. 2014). In consequence, soft X-ray brightness variations are solely due to different path lengths through the coronal plasma, sampling essentially the shape of the local bubble (Snowden et al. 1990; McCammon \& Sanders 1990).

The discovery of a soft X-ray shadow of the high galactic altitude Draco cloud (Snowden et al. 1991) led to a revision of the local hot bubble model. Moreover, Pietz et al. (1998) demonstrated that the soft X-ray background intensity distribution can be modeled quantitatively across the full sky adopting a simplified soft X-ray radiation transfer:

$I=I_{\text {local }}+I_{\text {distant }} \cdot \mathrm{e}^{-\bar{\sigma} \cdot N_{\mathrm{HI}}}$.

In addition to the local soft X-ray thermal emission, $I_{\text {local }}$ a much more luminous and slightly hotter thermal plasma component $I_{\text {distant }}$ fills a flattened Milky Way halo (scale heights $4.4 \mathrm{kpc}$, scale length $15 \mathrm{kpc}$; Pietz et al. 1998). This distant soft X-ray emission originates beyond the bulk of the neutral hydrogen (see Kalberla \& Kerp 2009, Fig. 11). Consequently, Snowden et al. (2000) released a catalog of 378 soft X-ray shadows associated with individual HI clouds.

Their Table 1 contains as shadow \#100 a four degree extended soft X-ray absorption feature positionally coincident with HRK 81.4-77.8. In Fig. 1 we show the $N_{\mathrm{HI}}$ map of HRK 81.4-77.8 (left-hand panel) and a slice across the whole cloud (right-hand panel). The ROSAT R12 count rates and the HI column density disclose a positional negative correlation. Accordingly, HRK 81.4-77.8 is physically coincident with the soft X-ray shadow \#100 of Snowden et al. (2000). The maximum HI column density of HRK 81.4-77.8 along that slice is $N_{\mathrm{HI}} \simeq 2.5 \times 10^{20} \mathrm{~cm}^{-2}$. Calculating the product of $N_{\mathrm{HI}}$ and the effective soft X-ray absorption cross section yields $\bar{\sigma} \cdot N_{\mathrm{HI}}=1.8$ for the ROSAT R12 band. An attenuation of about $83 \%$ of all soft X-ray emission originating beyond HRK 81.4-77.8 is expected (see Eq. (1)). In summary, towards the high column density portion of the cloud we observe dominantly $I_{\text {local }}$, while the soft X-ray shadow detection confirms by itself a localization of the cloud in front of the bulk of the Milky Way halo plasma. Pietz et al. (1998) determined that the scale height of the Milky
Way X-ray halo is about $4.4 \mathrm{kpc}$. Adopting a distance of $500 \mathrm{pc}$ about $50 \%$ of the halo plasma is located beyond HRK 81.4-77.8. Thus, the ROSAT soft X-ray shadow implies a nearby location of HRK 81.4-77.8 because of its significant depth. However, an upper distance limit of $500 \mathrm{pc}$ is compatible with the cloud's absorption feature within the uncertainties of the X-ray data.

Towards the deepest portions of the soft X-ray shadow of HRK 81.4-77.8 we observe dominantly $I_{\text {local }}$. This foreground emission is a measure for the minimum separation between the cloud and the Sun. Snowden et al. (2000) determined an $I_{\text {local }}$ count rate of only $(3.7 \pm 0.4) \times 10^{-4} \mathrm{cts} \mathrm{s}^{-1} \mathrm{arcmin}^{-2}$ in the ROSAT R12 energy band. This is quantitatively equal to the $I_{\text {local }}$ count rate observed towards MBM 12 (Snowden et al. $1993)$ with $(3.7 \pm 0.4) \times 10^{-4} \mathrm{cts} \mathrm{s}^{-1} \mathrm{arcmin}^{-2}$. However, MBM12 is located at a distance of $(360 \pm 30)$ pc (Andersson et al. 2002).

Because of systematic uncertainties in determining the absolute quantity of $I_{\text {local }}$ (Galeazzi et al. 2014; Puspitarini et al. 2014) we explore the error-range of this distance estimate. For this aim we inspect the local count rate towards

- the Draco cloud (Snowden et al. 2000 shadow \#116, $I_{\text {local }}=$ $(4.8 \pm 0.2) \times 10^{-4} \mathrm{cts} \mathrm{s}^{-1} \mathrm{arcmin}^{-2}$ at a distance between $463 \mathrm{pc}$ and $618 \mathrm{pc}$ Gladders et al. 1998) and

- IVC 135+54 (Snowden et al. 2000 shadow \#182, $I_{\text {local }}=$ $(5.8 \pm 0.2) \times 10^{-4} \mathrm{cts} \mathrm{s}^{-1} \operatorname{arcmin}^{-2}$ at $(395 \pm 95) \mathrm{pc}$ Benjamin et al. 1996).

Both $I_{\text {local }}$ count rates are in excess of that observed towards HRK 81.4-77.8. Implying that HRK 81.4-77.8 is most likely located in between MBM 12 and both IVCs within a distance bracket of $300 \mathrm{pc}$ to $500 \mathrm{pc}$. While the lower distance limit is affected by systematic uncertainties in evaluating $I_{\text {local }}$ (Galeazzi et al. 2014), the depth of the strong soft X-ray shadow sets a robust upper distance limit, which is of greater importance here for our discussion. Adopting this distance bracket we have to consider HRK 81.4-77.8 as a low-extinction, diffuse interstellar cloud located within the Milky Way's disk-halo interface. Thus, our result is in conflict with the $5 \mathrm{kpc}$ distance estimate of Camargo et al. (2015). Consequently, the stars of both stellar clusters might be significantly fainter and of much lower mass than initially assumed.

\subsection{Estimating the distance to HRK 81.4-77.8: Proper motion information towards Camargo 439}

Associated with the stellar cluster Camargo 439 are two proper motion measurements; the absolute length of the proper motion vectors are both about 6.2 marcsec $\mathrm{yr}^{-1}$ (see Camargo et al. 2015, Table 2). This proper motion corresponds to

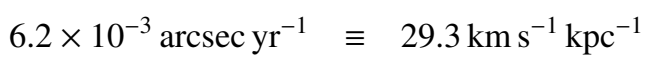




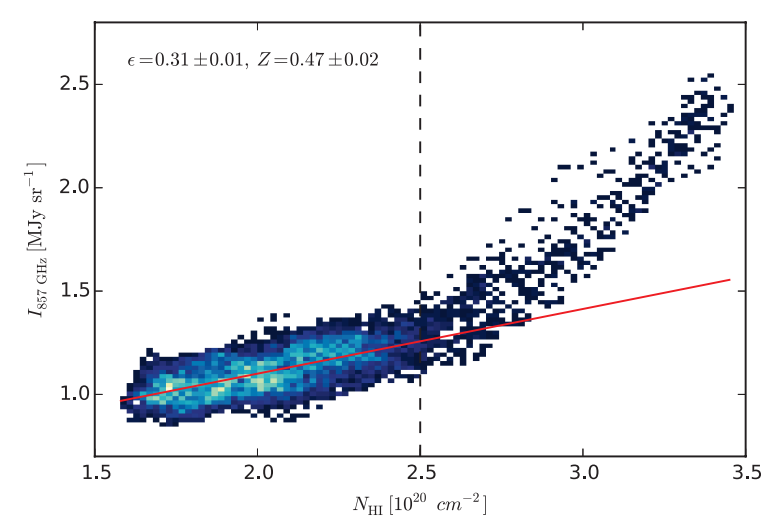

Fig. 2. $N_{\mathrm{HI}}$ versus $I_{\mathrm{FIR}} 857 \mathrm{GHz}$ correlation plot. The solid line represents a linear approximation (see Eq. (3)) to the data. Above $N_{\mathrm{HI}}=$ $2.5 \times 10^{20} \mathrm{~cm}^{-2}$ FIR excess emission is observed. This emission is attributed to the presence of additional molecular hydrogen regions not traced by $N_{\mathrm{HI}}$.

yielding $v_{\perp} \simeq 147 \mathrm{~km} \mathrm{~s}^{-1}$ at a distance of $5 \mathrm{kpc}$, as Camargo et al. (2015) assume. These very high tangential velocities are unexpected for young stellar clusters (Foster et al. 2015), suggesting that Camargo 439 needs to be extremely young to be detected as an entity because the two stellar proper motion vectors are oriented almost perpendicular to each other. Camargo et al. (2015) estimated an age of $2 \pm 1$ Myr for Camargo 439 which appears to be incompatible with the high proper motion. Moving for a period of $1 \mathrm{Myr}$ with 6 marcsec $^{-1} \mathrm{r}^{-1}$ would displace the stars by more than a degree from their birthplace. These numbers are inconsistent with the apparent overdensity of the stellar members of Camargo 439 (see Camargo et al. 2015, Fig. 2 left).

Adopting the hypothesis that Camargo 439 is physically associated with HRK 81.4-77.8 and consequently located at an upper distance limit of $400 \mathrm{pc}$ yields $v_{\perp} \simeq 11.7 \mathrm{~km} \mathrm{~s}^{-1}$. Even this value is in some conflict with actual studies of the stellar velocity dispersion of a young stellar cluster (Foster et al. 2015), but of the same order of magnitude as the turbulence broadened HI line width of the warm gas component of HRK 81.4-77.8 (Fig. 3).

\subsection{FIR emission of HRK 81.4-77.8}

Heiles et al. (1988) investigated the FIR, HI, and carbon monoxide (CO) properties of 26 isolated, degree-sized interstellar clouds. In the case of HRK 81.4-77.8 they did not report on CO (see Heiles et al. 1988, Table 2). Magnani et al. (2000) performed a southern hemisphere survey of the ${ }^{12} \mathrm{CO}(1 \rightarrow 0)$ transition. Their survey also does not comprise any information on the CO content of HRK 81.4-77.8 (see Magnani et al. 2000, Fig. 1).

The maximum HI column density of HRK 81.4-77.8 at GASS angular resolution of $14.4^{\prime}$ is $N_{\mathrm{HI}}=3.9 \times$ $10^{20} \mathrm{~cm}^{-2}$. This neutral hydrogen column density exceeds the molecule formation threshold value of $N_{\mathrm{HI}}=2.0 \times$ $10^{20} \mathrm{~cm}^{-2}$ (Planck Collaboration XXIV 2011). Above that value the FIR emissivity cannot be approximated by $N_{\mathrm{HI}}$ alone; additionally, we have to account for molecular hydrogen $N_{\mathrm{H}_{2}}$ because $I_{\mathrm{FIR}} \propto N_{\mathrm{H}}=N_{\mathrm{HI}}+2 \cdot N_{\mathrm{H}_{2}}$. Associated with HRK 81.4-77.8 we expect the presence of a detectable amount of molecular hydrogen from the FIR.

Correlating the Planck FIR $857 \mathrm{GHz}$ and the GASS HI data allows us to evaluate the gas-to-dust ratio and the amount of $N_{\mathrm{H}_{2}}$. Molecular gas, not quantitatively traced by neutral hydrogen, can be identified by an excess over the linear FIR-to- $N_{\mathrm{HI}}$ correlation. Figure 2 shows the $857 \mathrm{GHz}$ FIR emission versus the HI column

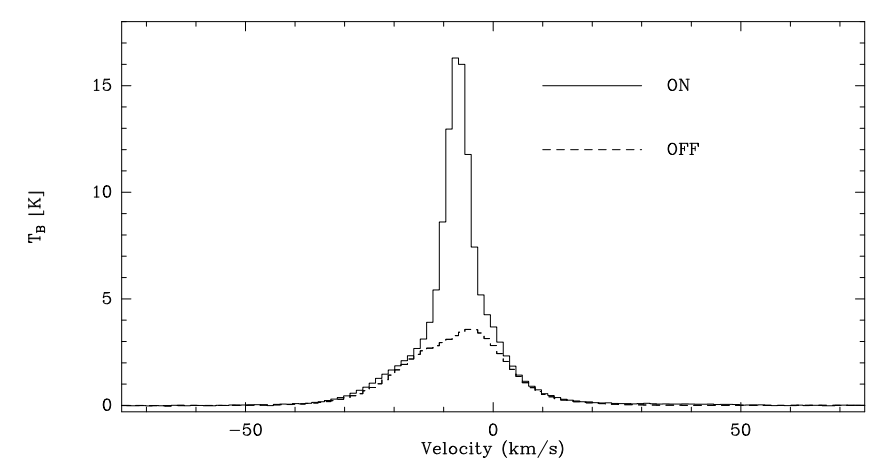

Fig. 3. Parkes GASS HI 21-cm line spectrum towards the stellar cluster Camargo 439 (solid line). Next to the bright narrow HI line a underlying broad $\mathrm{HI}$ emission line is visible. Offset from that position we extracted an $\mathrm{HI}$ spectrum of the unrelated $\mathrm{HI}$ emission (dashed line).

density. For the quantitative evaluation we smooth the Planck data to the lower angular resolution of GASS. The linear portion of the HI/FIR correlation is evaluated by

$I_{\mathrm{FIR}}=\epsilon \cdot N_{\mathrm{HI}}+Z$.

For the dust emissivity we find $\epsilon=(0.31 \pm 0.01) \times 10^{-20} \mathrm{MJy} \mathrm{sr}^{-1}$ and $Z=(0.47 \pm 0.02) \mathrm{MJy} \mathrm{sr}^{-1}$. The slope is compatible to the mean Galactic value between $\epsilon=0.3-0.7 \times 10^{-20} \mathrm{MJy} \mathrm{sr}^{-1} \mathrm{~cm}^{2}$ (Boulanger et al. 1996; Planck Collaboration XXIV 2011). This too is a strong argument for a nearby location of HRK 81.4-77.8, in addition to the ROSAT soft X-ray shadow. HRK 81.4-77.8 appears to be exposed to the same radiation field as a typical local Galactic interstellar cloud.

\subsection{Molecular gas fraction of HRK 81.4-77.8}

Although CO has not yet been observed in HRK 81.4-77.8, COdark molecular hydrogen may be present. Figure 2 shows that above $N_{\mathrm{HI}}=2.0 \times 10^{20} \mathrm{~cm}^{-2}$ the linear relation between dust and gas does not match quantitatively. Excess FIR emission is commonly attributed to the presence of molecular hydrogen $N_{\mathrm{H}_{2}}$ (Reach et al. 1998). An upper limit for $N_{\mathrm{H}_{2}}$ can be determined by attributing all FIR excess emission to $N_{\mathrm{H}_{2}}$. At a resolution of 14.4 arcmin we derive a peak column density for $\mathrm{H}_{2}$ of $N_{\mathrm{H}_{2}}=2.8 \times 10^{20} \mathrm{~cm}^{-2}$ from the FIR/HI correlation shown in Fig. 2. We chose for this scatter diagram a circular aperture centered at the HI peak with a radius of $2.7 \mathrm{deg}$. Consequently HRK 81.4-77.8 is dominantly an atomic cloud with a significant fraction of molecular gas. Locally a molecular fraction of up to $f=60 \%\left(f=\frac{2 \cdot N_{\mathrm{H}_{2}}}{N_{\mathrm{HI}}+2 \cdot N_{\mathrm{H}_{2}}}\right)$ is deduced. These regions are positionally coincident with cold neutral medium (CNM) gas.

To estimate the volume-filling factor of the molecular gas in HRK 81.4-77.8, which are below the angular resolution level of the Parkes telescope, we evaluate the FIR intensity contrast between the individual FIR peaks and their neighborhood at Planck's full angular resolution of $4.3 \mathrm{arcmin}$ at $857 \mathrm{GHz}$. On these angular scales, corresponding to physical sizes of about $0.5 \mathrm{pc}$ (at $400 \mathrm{pc}$ altitude), a variation in FIR brightness of up to a factor of two can be determined. This suggests, that the volume density is not expected to increase by more than an order of magnitude between linear scales of $0.5 \mathrm{pc}$ and $1.6 \mathrm{pc}$ (Parkes resolution). All gaseous properties of HRK 81.4-77.8 are consistent with normal high galactic latitude clouds. Moreover, the significant fraction of molecular gas can be considered typical for an infrared cirrus object (Gillmon \& Shull 2006). 


\subsection{HI properties of HRK 81.4-77.8}

Its extinction classifies HRK 81.4-77.8 as a diffuse cloud. It is host to a significant fraction of molecular gas of up to $60 \%$ of the total hydrogen column density. Most likely it is located within the disk-halo interface of the Milky Way Galaxy. A few high galactic latitude clouds at these altitudes have been studied so far, but disclosing very similar physical properties (Moritz et al. 1998; Weiß et al. 1999; Heithausen et al. 2001; Hernandez et al. 2013; Röhser et al. 2014; Lenz et al. 2015). The most significant difference is, however, the association of HRK 81.4-77.8 with two stellar clusters. If this association is a real one and not an accidental, this would have major implications for the formation of dwarf stars and brown dwarfs.

\subsubsection{Density perturbation in the warm neutral medium}

Figure 3 shows the HI spectrum towards Camargo 438 averaged across an area of a single GASS beam. It discloses a twocomponent HI spectrum. The peak brightness temperature is about $T_{\mathrm{B}}=17.7 \mathrm{~K}$ and the full width at half maximum (FWHM) is only $\Delta v_{\text {narrow }}(F W H M)=4.9 \mathrm{~km} \mathrm{~s}^{-1}$. This narrow line width corresponds to an upper limit for the kinetic temperature of $T_{\text {kin }}=550 \mathrm{~K}$. In such a cold cloud the velocity of sound is only $c_{\mathrm{S}}=2.7 \mathrm{~km} \mathrm{~s}^{-1}$.

The HI line profile displays a second HI line component that is dimmer but broader. Approximating simultaneously two Gaussian profiles to this HI spectrum yields almost the same Doppler velocity of the CNM $v_{\text {narrow }}=-6.9 \pm 0.1 \mathrm{~km} \mathrm{~s}^{-1}$ and a warm $v_{\text {broad }}=-7.2 \pm 0.1 \mathrm{~km} \mathrm{~s}^{-1}$ component. The line width of the warm neutral medium (WNM) is $\Delta v_{\text {broad }}(F W H M)=$ $22 \mathrm{~km} \mathrm{~s}^{-1}$. The column density of the broad component is $N_{\mathrm{HI}}(\mathrm{WNM})=1.8 \times 10^{20} \mathrm{~cm}^{-2}$ and about $30 \%$ higher than for the narrow line component $\left(N_{\mathrm{HI}}(\mathrm{CNM})=1.3 \times 10^{20} \mathrm{~cm}^{-2}\right)$.

Offset from HRK 81.4-77.8 we extracted an HI spectrum of the unrelated environmental HI gas. It is shown in Fig. 3 by the dashed line. This off-cloud HI spectrum fits the second broad HI component towards Camargo 438 nicely. This finding implies that the warm HI gas component ON Camargo 438 is the same as OFF to HRK 81.4-77.8.

Accordingly, we cannot attribute the whole WNM gas component observed towards Camargo 438 to HRK 81.4-77.8, but to the WNM gas layer of the Milky Way Galaxy. The similarity of the radial velocities of HRK 81.4-77.8 and the WNM argues, however, for a common origin of both. HRK 81.4-77.8 might be the result of a distortion in the velocity field (ram pressure) of the WNM initiating the molecular gas formation.

\subsubsection{Ram pressure}

Figure 4 shows the dynamics of the HI gas. Displayed are six brightness temperature maps of subsequent spectral channels. Starting at $v_{\mathrm{LSR}}=-12.78 \mathrm{~km} \mathrm{~s}^{-1}$ it is possible to see that even beyond the FIR emission of HRK 81.4-77.8 dynamically related HI emission is observed. We note that the displayed Doppler velocity panels sample the narrow (CNM) portion of the HI spectral line of HRK 81.4-77.8 (Fig. 3). The whole field of interest is filled with HI gas moving at comparable Doppler velocities and sharing similar properties with respect to spectral line width and brightness temperature to HRK 81.4-77.8. A remarkable rapid structural variation of the HI brightness temperature from channel to channel is obvious. With decreasing Doppler velocity we can identify a ring like structure in the
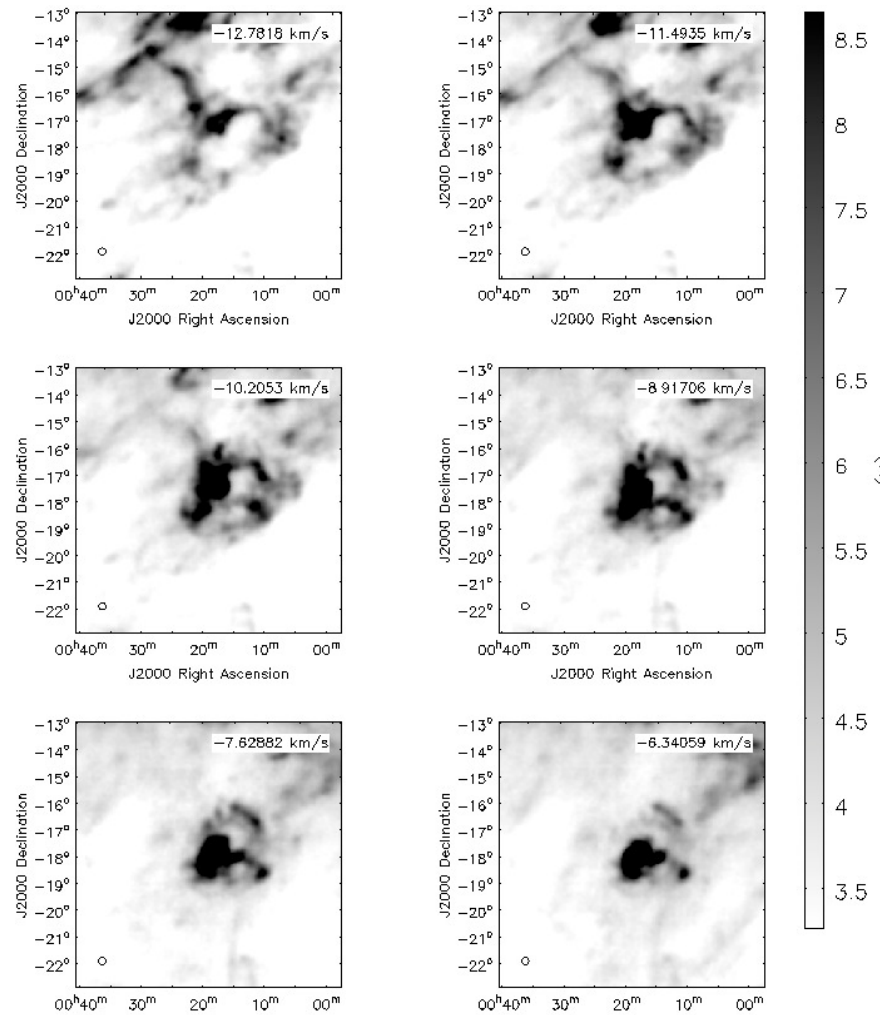

Fig. 4. HI 21-cm brightness temperature maps of six subsequent velocity channels. HRK 81.4-77.8 is located in the very center of the map. Note, that bright HI emission is detected across the whole field of interest, comparable in both, the brightness as well as in line width. HRK 81.4-77.8 is hence part of a much larger extended HI structure. Remarkable is the gas motion from top-left to HRK 81.4-77.8 in the very center in hand with the formation of a ring-like structure encircling HRK 81.4-77.8.

middle and bottom panels of Fig. 4. It encircles the FIR emission of HRK 81.4-77.8.

Across the extent of the FIR emission of HRK 81.4-77.8, Fig. 4 discloses a Doppler velocity gradient of at least $6.4 \mathrm{~km} \mathrm{~s}^{-1}$. This Doppler velocity gradient will cross the whole cloud within a few $10^{5} \mathrm{yr}$.

\section{Discussion and conclusions}

In this paper we determine an upper distance limit for HRK 81.4-77.8. This is an important piece of information considering the challenging proposal of Camargo et al. (2015) that the densest portions of this clouds are associated with star formation at an altitude of about $5 \mathrm{kpc}$ above the Galactic plane. The low extinction, the FIR emissivity, the implausible tangential velocities, and the soft X-ray shadow consistently argue for a location of HRK 81.4-77.8 at an altitude of between 300 and 500 pc. Its Doppler velocity classifies HRK $81.4-77.8$ as a low-velocity cloud with a two-phase medium. HRK 81.4-77.8 is continuously connected to neighboring $\mathrm{HI}$ gas showing up with comparable radial velocities and $\mathrm{HI}$ emission properties. In all aspects HRK 81.4-77.8 is a normal high galactic altitude cloud hosting some molecular regions. These molecular regions are of about $0.5 \mathrm{pc}$ extent and show up with a total hydrogen mass of $n_{\mathrm{H}}=n_{\mathrm{HI}}+2 \cdot n_{\mathrm{H}_{2}}$ of $M_{\mathrm{H}} \simeq 10 M_{\odot}$. The association with the young stellar clusters is unexpected (Camargo et al. 2015). Special in addition to HRK 81.4-77.8 is however the large scale radial velocity gradient, perhaps tracing a volume density/gas pressure 
situation out of any equilibrium between the WNM and CNM. This implies that the cloud might be in a transient state and not in an equilibrium state.

Figure 4 is suggestive for a ram-pressure interaction between the gas associated with HRK 81.4-77.8 and the disk-halo interface gas layers. This ram pressure might provide the necessary additional energy to trigger a WNM to CNM transition similar to the situation described by Röhser et al. (2014, 2016). Eventually, this will lead to the formation of molecular hydrogen even towards low column density clouds in the disk-halo interface, as reported by Gillmon \& Shull (2006), Planck Collaboration XXV (2011).

At the altitude of the disk-halo interface (about $400 \mathrm{pc}$; (Kalberla \& Kerp 2009), the Galactic radiation field is sufficiently thinned out (Haffner et al. 2009). Hence, the probability of detecting a significant fraction of molecular gas towards low column density diffuse clouds is high. That this is indeed the case, even in low-extinction diffuse interstellar clouds, is observationally confirmed by multiple observations via HI/FIR correlations and CO emission maps (Moritz et al. 1998; Weiß et al. 1999; Heithausen et al. 2001; Röhser et al. 2014; Lenz et al 2015). This holds true also on linear scales below one pc. Here, molecular structures have been observed as a common phenomenon towards the high Galactic latitude sky as reported by Heithausen (2007) and Gillmon \& Shull (2006).

When stars are really formed at high galactic altitudes, they will chemically and dynamically resemble their gaseous nursery. Smooth gradients in the chemical composition of dwarf halo stars and in their angular momentum distribution should be observed (Fuhrmann 1998; Nidever et al. 2014), but first and foremost we need to study the stellar population of Camargo 438 and 439 in much more detail.

Acknowledgements. The authors thank the Deutsche Forschungsgemeinschaft (DFG) for supporting the Effelsberg-Bonn HI Survey project under grant number KE 757/7-1 to -3 KE 757/9-1 and KE 757/11-1. D.L. and T.R. are members of the Bonn-Cologne Graduate School of Physics and Astronomy (BCGS). T.R. is a member of the International Max-Planck Research School (IMPRS) for Astronomy and Astrophysics at the Universities of Bonn and Cologne.

\section{References}

Andersson, B.-G., Idzi, R., Uomoto, A., et al. 2002, AJ, 124, 2164 Benjamin, R. A., Venn, K. A., Hiltgen, D. D., \& Sneden, C. 1996, ApJ, 464, 836 Boulanger, F., Abergel, A., Bernard, J.-P., et al. 1996, A\&A, 312, 256 Camargo, D., Bica, E., Bonatto, C., \& Salerno, G. 2015, MNRAS, 448, 1930 Foster, J. B., Cottaar, M., Covey, K. R., et al. 2015, ApJ, 799, 136 Frisch, P. C., \& York, D. G. 1983, ApJ, 271, L59
Fuhrmann, K. 1998, A\&A, 338, 161

Galeazzi, M., Chiao, M., Collier, M. R., et al. 2014, Nature, 512, 171 Gillmon, K., \& Shull, J. M. 2006, ApJ, 636, 908

Gladders, M. D., Clarke, T. E., Burns, C. R., et al. 1998, ApJ, 507, L161

Green, G. M., Schlafly, E. F., Finkbeiner, D. P., et al. 2015, ApJ, 810, 25

Haffner, L. M., Dettmar, R.-J., Beckman, J. E., et al. 2009, Rev. Mod. Phys., 81, 969

Heiles, C., Reach, W. T., \& Koo, B.-C. 1988, ApJ, 332, 313

Heithausen, A. 2007, in SINS - Small Ionized and Neutral Structures in the Diffuse Interstellar Medium, eds. M. Haverkorn, \& W. M. Goss, ASP Conf. Ser., 365, 177

Heithausen, A., Weiß, A., Kerp, J., \& Fritz, T. 2001, ApJ, 561, 238

Hernandez, A. K., Wakker, B. P., Benjamin, R. A., et al. 2013, ApJ, 777, 19

Kalberla, P. M. W., \& Haud, U. 2015, A\&A, 578, A78

Kalberla, P. M. W., \& Kerp, J. 2009, ARA\&A, 47, 27

Kalberla, P. M. W., McClure-Griffiths, N. M., Pisano, D. J., et al. 2010, A\&A, 521, A 17

Kennicutt, R. C., \& Evans, N. J. 2012, ARA\&A, 50, 531

Lallement, R., Vergely, J.-L., Valette, B., et al. 2014, A\&A, 561, A91

Lenz, D., Kerp, J., Flöer, L., et al. 2015, A\&A, 573, A83

Magnani, L., Blitz, L., \& Mundy, L. 1985, ApJ, 295, 402

Magnani, L., Hartmann, D., Holcomb, S. L., Smith, L. E., \& Thaddeus, P. 2000, ApJ, 535, 167

McCammon, D., \& Sanders, W. T. 1990, ARA\&A, 28, 657

McClure-Griffiths, N. M., Pisano, D. J., Calabretta, M. R., et al. 2009, ApJS, 181,398

McGehee, P. M. 2008, Star Formation and Molecular Clouds at High Galactic Latitude, ed. B. Reipurth, 813

Moritz, P., Wennmacher, A., Herbstmeier, U., et al. 1998, A\&A, 336, 682

Nidever, D. L., Bovy, J., Bird, J. C., et al. 2014, ApJ, 796, 38

Paresce, F. 1984, AJ, 89, 1022

Pietz, J., Kerp, J., Kalberla, P. M. W., et al. 1998, A\&A, 332, 55

Planck Collaboration IX. 2014, A\&A, 571, A11

Planck Collaboration XXIV. 2011, A\&A, 536, A24

Planck Collaboration XXV. 2011, A\&A, 536, A25

Puspitarini, L., Lallement, R., Vergely, J.-L., \& Snowden, S. L. 2014, A\&A, 566, A13

Reach, W. T., Wall, W. F., \& Odegard, N. 1998, ApJ, 507, 507

Röhser, T., Kerp, J., Winkel, B., Boulanger, F., \& Lagache, G. 2014, A\&A, 564, A71

Röhser, T., Kerp, J., Ben Bekhti, N., \& Winkel, B. 2016, A\&A, submitted

Schlafly, E. F., \& Finkbeiner, D. P. 2011, ApJ, 737, 103

Schlegel, D. J., Finkbeiner, D. P., \& Davis, M. 1998, ApJ, 500, 525

Snowden, S. L., \& Schmitt, J. H. M. M. 1990, Ap\&SS, 171, 207

Snowden, S. L., Cox, D. P., McCammon, D., \& Sanders, W. T. 1990, ApJ, 354 211

Snowden, S. L., Mebold, U., Hirth, W., Herbstmeier, U., \& Schmitt, J. H. M. 1991, Science, 252, 1529

Snowden, S. L., McCammon, D., \& Verter, F. 1993, ApJ, 409, L21

Snowden, S. L., McCammon, D., Burrows, D. N., \& Mendenhall, J. A. 1994, ApJ, 424, 714

Snowden, S. L., Egger, R., Freyberg, M. J., et al. 1997, ApJ, 485, 125

Snowden, S. L., Freyberg, M. J., Kuntz, K. D., \& Sanders, W. T. 2000, ApJS, 128,171

Weiß, A., Heithausen, A., Herbstmeier, U., \& Mebold, U. 1999, A\&A, 344, 955

Wilms, J., Allen, A., \& McCray, R. 2000, ApJ, 542, 914

Wright, E. L., Eisenhardt, P. R. M., Mainzer, A. K., et al. 2010, AJ, 140, 1868 